\title{
PARADISE - Eine Matlab-Toolbox für Entwurf und Analyse robuster Regelungen
}

\author{
Wolfgang Sienel und Tilman Bünte, Oberpfaffenhofen
}

Dr.-Ing. Wolfgang Sienel ist wissenschaftlicher Mitarbeiter im DLR-Institut für Robotik und Systemdynamik (FF-DR). Seine Arbeitsgebiete sind Analyse und Entwurf von robusten Regelungssystemen, die Entwicklung von Softwaretools für die robuste Regelung, sowie die Anwendung von Regelung im fahrzeugdynamischen Bereich.

Adresse: DLR, Institut für Robotik und Systemdynamik, Postfach 1116, 82230 Weßling, Email: Wolfgang.Sienel@dlr.de

Dipl.-Ing. Tilman Bünte ist Doktorand im DLR-Institut FF-DR. Arbeitsgebiete sind die robuste Regelungstechnik und deren Anwendung auf aktive Fahrzeuglenkung.

Adresse: wie oben, Email: Tilman.Buente@dlr.de

\section{Kurzfassung}

Entwurfs- und Analyseverfahren für die parametrisch orientierte robuste Regelungstechnik benötigen aufwendige symbolische und numerische Berechnungen, die nur in einfachen Fällen noch von Hand ausgeführt werden können. Mit PARADISE (PArametric Robustness Analysis and Design Interactive Software Environment) werden verschiedene Methoden der robusten Regelung für den effizienten Einsatz im Rechner zur Verfügung gestellt. Zu den implementierten Methoden gehören unter anderem das Parameterraumverfahren, der Entwurf in der Invarianzebene, die Berechnung des Stabilitätsradius und die Konstruktion von Wertemengen. Anhand des Entwurfsbeispiels einer automatischen Spurführung für ein Fahrzeug wird der Einsatz der neuen Toolbox PARADISE demonstriert.

\section{PARADISE - A Matlab-toolbox for robust parametric control}

Design and analysis methods for parameter oriented robust control require extensive symbolic and numeric computations which only for simple cases can be carried out using paper and pencil. PARADISE (PArametric Robustness Analysis and Design Interactive Software 
Environment) offers several methods for robust control in an efficient manner. Among the implemented techniques there are the parameter space approach, design in an invariance plane, computation of stability radii, and construction of value sets. The use of the toolbox PARADISE is demonstrated by a controller design of an automatic tracking control for a vehicle.

\section{$1 \quad$ Einführung}

Die Grundlage der robusten Regelung, wie sie hier betrachtet wird, ist ein physikalisch motiviertes Modell der Strecke, wobei die unsicheren Streckenparameter $\boldsymbol{q}=\left[\begin{array}{llll}q_{1} & q_{2} & \ldots & q_{\ell}\end{array}\right]^{T}$ mit in die Streckenbeschreibung eingehen. Dabei darf die Abhängigkeit der Koeffizienten des charakteristischen Polynoms von diesen Parametern auch multilinearer oder sogar polynomialer Art sein. Im allgemeinen sind die unsicheren Parameter voneinander unabhängig und werden durch untere und obere Schranken begrenzt. Der Betriebsbereich

$$
Q:=\left\{\boldsymbol{q} \mid q_{i} \in\left[q_{i}^{-} ; q_{i}^{+}\right], i=1,2, \ldots, \ell\right\}
$$

wird dann durch ein Hyperrechteck („Q-Box“) beschrieben. Beispiel hierfür ist das Modell eines Kraftfahrzeugs, wobei die wesentlichen unsicheren Parameter die Masse $m$, die Fahrgeschwindigkeit $v$ und der Kraftschlußkoeffizient $\mu$ sind. Alle diese Parameter können innerhalb ihrer Grenzen variieren. Eine Modellbeschreibung, z.B. eine linearisierte Zu-

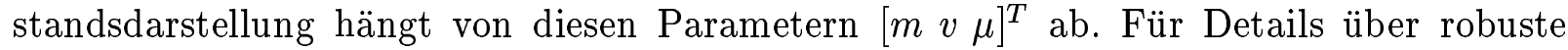
Regelung sei der verehrte Leser auf [1] verwiesen.

Bei den meisten regelungstechnischen Anwendungen im Fahrzeug steht die Fahrgeschwindigkeit $v$ durch Messung zur Verfügung. Die Aufgabe des Ingenieurs besteht nun darin, einen Regler zu entwerfen, der für den gesamten Bereich der unsicheren Parameter $\boldsymbol{q}=[m \mu]^{T}$ die Spezifikationen für den geschlossenen Regelkreis erfüllt, eventuell durch Betriebsanpassung der Reglerparameter unter geeigneter Ausnutzung des vorhandenen Meßsignals für die Geschwindigkeit.

Diese physikalisch motivierte Vorgehensweise steht im Gegensatz zu anderen Methoden der robusten Regelung, in denen ein nominales Modell der Strecke gegeben ist und eine in der Norm beschränkte Abweichung vom Nominalmodell angenommen wird. Diese Modellannahmen sind mathematisch und rechentechnisch attraktiver, da sie auf einer einheitlichen Problemstellung basieren. Allerdings besitzt dieses mathematische Modell wenig Bezug zur tatsächlichen physikalischen Strecke. Zugleich werden häufig Überabschätzungen getroffen, was zu konservativen Ergebnissen führt. Typischerweise sind die daraus resultierenden Regler von hoher Ordnung, weitere Entwurfskriterien können in der Regel nicht berücksichtigt werden. Hierzu bekannte Verfahren sind die $\mu$-Synthese und das $\mathcal{H}^{\infty}$-Entwurfsverfahren, die ebenfalls in Form von Matlab-Toolboxen verfügbar sind $[2,3]$.

Die meisten Verfahren der Robusten Regelung ${ }^{1}$ sind hinlänglich in der Literatur bekannt.

\footnotetext{
${ }^{1} \mathrm{Ab}$ hier bezieht sich der Begriff der „Robusten Regelung“ nur noch auf den parametrischen Ansatz.
} 
Meist benötigen diese Verfahren aufwendige numerische und symbolische Berechnungen, was viele Entwurfsingenieure vor deren Anwendung abschreckt. Es ist das Ziel von PARADISE, diese Methoden in einer komfortablen und flexiblen Weise zur Verfügung zu stellen.

Die Schwierigkeiten bei der Behandlung parametrischer Modelle sollen an dem folgenden Beispiel aus [1] illustriert werden:

Beispiel 1.1 Gegeben sei das unsichere charakteristische Polynom

$$
p(s, \boldsymbol{q})=k_{1} g+k_{2} g s+\left(\left(m_{L}+m_{C}\right) g+k_{1} \ell_{R}\right) s^{2}+k_{2} \ell_{R} s^{3}+\ell_{R} m_{C} s^{4}
$$

einer geregelten Verladebrücke mit den unsicheren Parametern Lastmasse $m_{L}$, Masse der Laufkatze $m_{C}$, Seillänge $\ell_{R}$ und den Reglerparametern $k_{1}, k_{2}$. Die Stabilität kann anhand der Hurwitzbedingungen $\left(a_{i}\right.$ ist jeweils der Koeffizient $\mathrm{zu} s^{i}$ )

$$
\begin{aligned}
a_{0}>0 & \Rightarrow k_{1}>0 \\
a_{1}>0 & \Rightarrow k_{2}>0 \\
a_{2}>0 & \Rightarrow k_{1}>-\left(m_{L}+m_{C}\right) g / \ell_{R} \\
a_{3}>0 & \Rightarrow k_{2}>0 \\
\Delta_{3}=k_{2}^{2} \ell_{R} m_{L} g & \Rightarrow k_{2} \neq 0
\end{aligned}
$$

untersucht werden, wobei $\Delta_{3}$ die vorletzte Hurwitzdeterminante ist. Das System erweist sich für beliebige positive Werte von $k_{1}$ und $k_{2}$ als stabil.

Bei technischen Systemen genügt Hurwitzstabilität alleine meist nicht den Anforderungen. So wird eine bestimmte Einschwingzeit gewährleistet, wenn sämtliche Eigenwerte zur linken einer verschobenen imaginären Achse $v \mapsto s+\sigma_{0}, \sigma_{0} \in \mathbb{R}^{+}$, liegen. Das transformierte charakteristische Polynom lautet dann

$$
p(v, \boldsymbol{q})=a_{0}^{*}+a_{1}^{*} v+a_{2}^{*} v^{2}+a_{3}^{*} v^{3}+a_{4}^{*} v^{4}
$$

mit

$$
\begin{aligned}
& a_{0}^{*}=k_{1} g-k_{2} g \sigma_{0}+\left[\left(m_{L}+m_{C}\right) g+k_{1} \ell_{R}\right] \sigma_{0}^{2}-k_{2} \ell_{R} \sigma_{0}^{3}+\ell_{R} m_{C} \sigma_{0}^{4} \\
& a_{1}^{*}=k_{2} g-2\left[\left(m_{L}+m_{C}\right) g+k_{1} \ell_{R}\right] \sigma_{0}+3 k_{2} \ell_{R} \sigma_{0}^{2}-4 \ell_{R} m_{C} \sigma_{0}^{3} \\
& a_{2}^{*}=\left(m_{L}+m_{C}\right) g+k_{1} \ell_{R}-3 k_{2} \ell_{R} \sigma_{0}+6 \ell_{R} m_{C} \sigma_{0}^{2} \\
& a_{3}^{*}=k_{2} \ell_{R}-4 \ell_{R} m_{C} \sigma_{0} \\
& a_{4}^{*}=\ell_{R} m_{C}
\end{aligned}
$$

Um zu überprüfen, ob der Realteil der Nullstellen von (1) kleiner als $\sigma_{0}$ ist, kann die Hurwitzstabilität des transformierten Polynoms (2) untersucht werden. Während die Hurwitzstabilität des Polynoms (1) noch sehr einfach bestimmt werden konnte, gestaltet sich die Überprüfung des transformierten Polynoms wegen der komplizierteren Parameterabhängigkeiten in den Koeffizientenfunktionen sehr viel schwieriger. 
Dieses Beispiel zeigte einige Schwierigkeiten auf, die sich bei der Behandlung parametrischer Modelle ergeben können. Durch Transformationen und andere Operationen können sich die Systemgleichungen erheblich verkomplizieren, was bei Ausführung von Hand in der Regel zu äußerst aufwendigen Rechnungen führt. Deshalb können die Methoden der robusten Regelung für parametrische Modelle nur mit entsprechenden computergestützten Werkzeugen effizient angewandt werden. Die Interaktion des Entwurfsingenieurs mit diesem Werkzeug sollte sich dabei möglichst nur auf die Eingaben, die Wahl der entsprechenden Methode und der Interpretation des Ergebnisses beschränken. Sämtliche symbolischen und numerischen Operationen, wie in dem obigen Beispiel, sollten verborgen im Hintergrund ablaufen.

Wie bereits bemerkt, ist Hurwitzstabilität alleine oft nicht ausreichend, um befriedigendes Systemverhalten zu garantieren. Deshalb wird das zulässige Eigenwertgebiet weiter eingeschränkt. Eine Reihe von Spezifikationen für den geschlossenen Regelkreis können unmittelbar in bezug auf die Eigenwertlage formuliert werden, wie z.B. Dämpfung und Bandbreite. Andere Anforderungen hingegen müssen erst in ihre Bedeutung für die Eigenwertlage übersetzt werden, etwa die Einschwingzeit. Bild 1 a)-c) zeigt einige sogenannte Basiselemente für die Berandung des zulässigen Eigenwertgebiets. Diese Beispiele stehen für a) maximale Einschwingzeit, b) minimale Dämpfung und c) Bandbreitenbegrenzung. Die nicht schraffierte Seite markiert das zulässige Eigenwertgebiet. Sollen mehrere Spezifikationen gleichzeitig erfüllt werden, ist die Schnittmenge dieser Eigenwertgebiete zu bilden (d)). Das zulässige Eigenwertgebiet wird mit $\Gamma$ bezeichnet, seine Berandung mit $\partial \Gamma$. Ein geschlossener Regelkreis heißt $\Gamma$-stabil, wenn alle seine Eigenwerte innerhalb des Gebietes $\Gamma$ liegen. Sämtliche der im nächsten Abschnitt vorgestellten Methoden können auf Hurwitzwie auch auf $\Gamma$-Stabilität angewandt werden.

\section{Implementierte Methoden}

Die allgemeine Aufgabe ist der Entwurf eines Reglers, der das System für den gegebenen Betriebsbereich $Q$ robust stabilisiert. Da es im allgemeinen kaum möglich ist, einen solchen Regler auf Anhieb zu finden, kann man folgenden praktikablen Weg gehen:

1. Wähle eine endliche Anzahl von Repräsentanten des Betriebsbereichs $Q$, z.B. signifikante Punkte wie die Ecken des Betriebsbereichs und versuche, einen Regler zu finden, der diese Repräsentanten simultan stabilisiert. Diese Entwurfsstrategie kann auch auf hochgradig nichtlineare Systeme angewandt werden, für die lediglich eine endliche Anzahl linearisierter Modelle bei unterschiedlichen Betriebspunkten zur Verfügung stehen (Multimodell-Ansatz). Ein typisches Beispiel hierfür ist die Flugregelung, die sich im allgemeinen auf die Betrachtung einer endlichen Zahl von Flugfällen beschränkt.

2. Überprüfe die Stabilität des geschlossenen Regelkreises für das gesamte Kontinuum des Betriebsbereichs mit dem im 1. Schritt gefundenen Regler. Falls dieser Test 
negativ ausfällt, werden für die Wiederholung von Schritt 1 neue, geeignete Repräsentanten hinzugenommen.

Die meisten der Verfahren, die gegenwärtig in einer ersten Version der Toolbox PARADISE implementiert werden, sind in [1] beschrieben. Es folgt eine kurze Übersicht über diese Verfahren.

\subsection{Das Parameterraumverfahren}

Das Parameterraumverfahren kann verwendet werden, um Stabilitätsgrenzen in einem zweidimensionalen Unterraum des Parameterraums ${ }^{2}$ darzustellen. Es basiert auf dem Grenzüberschreitungssatz von Frazer und Duncan [4].

Man betrachte das unsichere charakteristische Polynom $p\left(s, t_{1}, t_{2}\right)$, welches nur von den beiden Parametern $t_{1}$ und $t_{2}$ abhängt. Diese beiden Parameter dürfen auch in polynomialer Weise in das Polynom eingehen. Für einen Punkt $\sigma+j \omega$ auf der $\Gamma$-Berandung $\partial \Gamma$ hat das charakteristische Polynom eine Nullstelle, wenn die beiden Gleichungen

$$
\begin{aligned}
& \operatorname{Re} p\left(\sigma+\mathrm{j} \omega, t_{1}, t_{2}\right)=0 \\
& \operatorname{Im} p\left(\sigma+\mathrm{j} \omega, t_{1}, t_{2}\right)=0
\end{aligned}
$$

gleichzeitig erfüllt sind. Die Ermittlung aller Lösungen $\left\{\left(t_{1}, t_{2}\right) \mid p\left(\sigma+\mathrm{j} \omega, t_{1}, t_{2}\right)=0, \sigma+\right.$ $\mathrm{j} \omega \in \partial \Gamma\}$ entlang eines Rasters auf $\partial \Gamma$ in der Eigenwertebene liefert die Abbildung von $\partial \Gamma$ in den Parameterraum $\left(t_{1}, t_{2}\right)$. Details zum Parameterraumverfahren sind in $[1,5] \mathrm{zu}$ finden.

Die Parameter $t_{1}$ und $t_{2}$ können unsichere Parameter der Strecke und/oder noch zu bestimmende Reglerparameter sein. Im Fall reiner Streckenparameter kann das Verfahren zur Robustheitsanalyse verwendet werden, d.h. das Sytem ist stabil, wenn ein beliebiger Betriebspunkt $\boldsymbol{q} \in Q$ gefunden werden kann, der $\Gamma$-stabil ist und keine der $\Gamma$-Stabilitätsgrenzen die $Q$-Box schneidet.

Im Fall von reinen Reglerparametern kann das Parameterraumverfahren für den Reglerentwurf herangezogen werden: In einem ersten Schritt werden die $\Gamma$-Stabilitätsgrenzen in einer zweidimensionalen Ebene innerhalb des Raums der Reglerparameter berechnet. Die $\Gamma$ - Stabilitätsgrenzen unterteilen die Reglerparameterebene in eine endliche Anzahl von Gebieten. Wenn sich ein beliebiger Punkt dieser Ebene als $\Gamma$-stabil herausstellt (was sich unmittelbar anhand des zugehörigen charakteristischen Polynoms ermitteln läßt), dann bedeutet dies $\Gamma$-Stabilität für das gesamte Gebiet, zu dem dieser Punkt gehört. Dies ist eine Eigenschaft des bereits erwähnten Grenzüberschreitungssatzes.

Bei unsicheren Strecken wird entsprechend der oben vorgestellten Strategie zunächst eine Anzahl von Repräsentanten ausgewählt und die zugehörigen Gebiete der $\Gamma$-stabilisierenden

\footnotetext{
${ }^{2}$ Der Parameterraum ist die Menge der Vektoren, dessen Elemente die betrachteten Parameter sind, z.B. unsichere Streckenparameter und Reglerparameter.
} 
Regler in der Reglerparameterebene bestimmt. Regler aus der Schnittmenge all dieser Gebiete $\Gamma$-stabilisieren simultan zumindest die ausgewählten Repräsentanten. Nachdem man einen Regler aus dieser Schnittmenge ausgewählt hat, muß eine anschließende Robustheitsanalyse durchgeführt werden, um Robustheit für den gesamten Betriebsbereich sicherzustellen. Falls die unsichere Strecke sich nicht als robust $\Gamma$-stabilisiert erweist, kann in der nächsten Iteration ein oder mehrere Repräsentanten aus dem $\Gamma$-instabilen Parametergebiet hinzugenommen werden.

Auch die Wahl einer aus einem veränderlichen Streckenparameter und einem Reglerparameter gemischten Parameterebene kann sinnvoll sein. Dann kann man mittels des Parameterraumverfahrens die Anpassung (gain scheduling) von Reglerparametern mit veränderlichen Betriebsparametern vornehmen [6].

\subsection{Entwurf in einer Invarianzebene}

Dieses Verfahren $[1,7]$ zum Entwurf von linearen Zustandsrückführungen basiert auf dem Parameterraumverfahren. Für eine nominale Strecke wird ein $m$-dimensionaler Querschnitt (Unterraum) im Raum der Reglerparameter so bestimmt, daß mit der Veränderung von Reglerparametern innerhalb dieses Unteraums genau $m$ Eigenwerte des geschlossenen Regelkreises verschoben werden. Für die praktische Anwendung ist $m=2$ und Unterräume mit der beschriebenen Eigenschaft heißen Invarianzebenen. So können schrittweise jeweils zwei Eigenwerte gleichzeitig verschoben werden, bis sämtliche Eigenwerte innerhalb des zulässigen $\Gamma$-Stabilitätsgebietes liegen.

Für eine nominale Regelstrecke $\dot{\boldsymbol{x}}=\boldsymbol{A} \boldsymbol{x}+\boldsymbol{b} u$ basiert die Ermittlung dieser Ebenen auf Ackermanns Formel [1]:

Satz 2.1 Ackermann

Für ein steuerbares System $(\boldsymbol{A}, \boldsymbol{b})$ werden mit dem Rückführvektor

$$
\boldsymbol{k}^{T}=\boldsymbol{e}^{T} p(\boldsymbol{A})
$$

mit

$$
\boldsymbol{e}^{T}=\left[\begin{array}{llll}
0 & 0 & \ldots & 1
\end{array}\right]\left[\begin{array}{lllll}
\boldsymbol{b} & \boldsymbol{A} \boldsymbol{b} & \boldsymbol{A}^{2} \boldsymbol{b} & \ldots & \boldsymbol{A}^{n-1} \boldsymbol{b}
\end{array}\right]^{-1}
$$

die Eigenwerte von $\boldsymbol{A}-\boldsymbol{b}^{T}$ durch die Wurzeln des Polynoms $p(s)$ vorgegeben.

Anhand dieses Satzes läßt sich nun der Rückführvektor $\boldsymbol{k}$ in $\operatorname{der}\left(\kappa_{a}, \kappa_{b}\right)$-Invarianzebene durch

$$
\boldsymbol{k}^{T}=\left[\kappa_{a} \kappa_{b}\right]\left[\begin{array}{l}
\boldsymbol{e}^{T} h(\boldsymbol{A}) \\
\boldsymbol{e}^{T} h(\boldsymbol{A}) \cdot \boldsymbol{A}
\end{array}\right]
$$

angeben, wobei das Polynom $h(s)$ die $n-2$ Nullstellen ( $n=$ Systemordnung) des charakteristischen Polynoms $p(s)=\operatorname{Det}(s \boldsymbol{I}-\boldsymbol{A})=t(s) \cdot h(s)$ repräsentiert, die durch die Rückführung 
$\boldsymbol{k}$ nicht verschoben werden sollen [1]. Durch die Rückführung werden lediglich die Nullstellen von $t(s)$ verschoben.

Diese Vorgehensweise kann unmittelbar auf ein nominales System angewandt werden. Handelt es sich jedoch um ein unsicheres System, so kann die Invarianzebene jeweils zunächst nur für einen nominalen Betriebspunkt ermittelt werden und die $\Gamma$-Stabilitätsgrenzen werden dann für die Repräsentanten in dieser Invarianzebene berechnet. Natürlich führt dies dazu, daß im allgemeinen bei einer so berechneten Rückführung alle Eigenwerte verschoben werden, sobald man nicht mehr den nominalen Betriebspunkt betrachtet. Wird als nominaler Betriebspunkt der Mittelpunkt der Q-Box zur Ermittlung der Invarianzebene gewählt, so ist jedoch in den meisten praktischen Fällen die Verschiebung der restlichen Eigenwerte nur geringfügig. Es empfiehlt sich, dieses Verfahren iterativ anzuwenden und in jedem Schritt die beiden kritischsten Eigenwerte in Richtung des gewünschten Polgebiets zu verschieben. In jedem Schritt sollte die gewählte Verstärkung nicht zu hoch gewählt werden. Auch hier gilt wieder, daß das Ergebnis mit einer Robustheitsanalyse für das Kontinuum des gesamten Betriebsbereichs verifiziert werden muß.

Der beispielhafte Entwurf für automatische Spurführung von Fahrzeugen in einer Invarianzebene wird in $[1,8]$ und auch anhand eines Anwendungsbeispiels von PARADISE in Kapitel 4 gezeigt.

\subsection{Wertemengenkonstruktion und Baumstrukturierte Zerlegung}

In Erweiterung des Mikhailov-Ortskurvenverfahrens [9] wird für charakteristische Polynome der sogenannte Nullauschlußsatz formuliert:

Satz 2.2 Das unsichere Polynom $p(s, \boldsymbol{q})$ ist für den Betriebsbereich $Q$ genau dann robust $\Gamma$-stabil, wenn

1. ein beliebiger Betriebspunkt $\boldsymbol{q}_{0} \in Q$ existiert, so daß $p\left(s, \boldsymbol{q}_{0}\right) \Gamma$-stabil ist und

2. die Wertemengen

$$
\mathcal{P}(s, Q):=\{p(s, \boldsymbol{q}) \mid \boldsymbol{q} \in Q\}
$$

für alle Frequenzen $s \in \partial \Gamma$ den Ursprung ausschließen.

Die Konstruktion von Wertemengen vereinfacht sich erheblich, wenn das System eine sogenannte Baumstruktur besitzt, d.h. wenn das charakteristische Polynom als Summe bzw. Produkt von Subpolynomen dargestellt werden kann, die keine gemeinsamen unsicheren Parameter besitzen. Wenn die Anzahl der unsicheren Parameter in diesen Subpolynomen niedrig ist, können ihre Wertemengen einfach konstruiert werden. Die Wertemenge des 
Polynoms $p(s, \boldsymbol{q})$ ergibt sich dann aus Summen und Produkten der Wertemengen der Subpolynome entsprechend der Baumstruktur von $p(s, \boldsymbol{q})$. Auf diese Weise können sogar Systeme mit einer großen Anzahl unsicherer Parameter sehr schnell auf ihre robuste $\Gamma$-Stabilität untersucht werden $[1,10]$.

Als Beispiel wird nun wieder das Polynom (1), diesmal in der Form

$$
p(s, \boldsymbol{q})=\left(s^{2} \ell_{R}+g\right)\left(k_{1}+k_{2} s+m_{C} s^{2}\right)+m_{L} g s^{2}
$$

betrachtet. Die Wertemengen von $s^{2} \ell_{R}+g, k_{1}+k_{2} s+m_{C} s^{2}$ und $m_{L} g s^{2}$ können für einen festen Punkt $s=\sigma+\mathrm{j} \omega$ auf $\partial \Gamma$ getrennt konstruiert werden, da sie keine gemeinsamen unsicheren Parameter enthalten. Die resultierenden Wertemengen müssen lediglich miteinander multipliziert und addiert werden. Auf diese Weise genügt es, ein eindimensionales Raster entlang $\partial \Gamma \mathrm{zu}$ überprüfen anstatt eines fünfdimensionalen Rasters im Raum der unsicheren Parameter $\ell_{R}, m_{C}, m_{L}, k_{1}$ und $k_{2}$.

Eine Erweiterung dieses Verfahrens erlaubt es, das sogenannte Stabilitätsprofil zu konstruieren, d.h. die Begrenzung der Wurzelmenge des unsicheren charakteristischen Polynoms aus der „Sicht“ des Punktes $s=+\infty$ [11]. Das Stabilitätsprofil gibt unmittelbar Auskunft über Robustheit und Stabilitätsreserve.

\subsection{Entwurf durch Kontraktion eines Polgebiets}

Allgemein ist die Menge der Reglerparametervektoren $\boldsymbol{k}$, für die das Polynom

$$
p(s, \boldsymbol{q}, \boldsymbol{k})=a_{0}(\boldsymbol{q}, \boldsymbol{k})+\ldots+a_{n-1}(\boldsymbol{q}, \boldsymbol{k}) s^{n-1}+a_{n}(\boldsymbol{q}, \boldsymbol{k}) s^{n}
$$

Hurwitz-stabil ist (im Fall von $\Gamma$-Stabilität müssen die transformierten Bedingungen bzgl. des gewählten $\Gamma$-Gebietes betrachtet werden), begrenzt durch die drei Hyperflächen

$$
\begin{array}{r}
a_{0}(\boldsymbol{q}, \boldsymbol{k})=0 \\
a_{n}(\boldsymbol{q}, \boldsymbol{k})=0 \\
\Delta_{n-1}(\boldsymbol{q}, \boldsymbol{k})=0
\end{array}
$$

Die ersten beiden dieser Hyperflächen stehen für reelle Grenzen in der Eigenwertebene bei Null bzw. im Unendlichen, die dritte (Verschwinden der vorletzten Hurwitzdeterminanten) steht für die konjugiert komplexe Stabilitätsgrenze (imaginäre Achse). In einer hinreichend kleinen Umgebung dieser Berandungen gilt: Je größer der Betrag des Funktionswertes von $a_{0}, a_{n}$ bzw. $\Delta_{n-1}$ ist, um so weiter enfernt von der entsprechenden Stabilitätsgrenze befindet sich das System. Deshalb entspricht ein größerer Abstand von diesen Hyperflächen auch einem größeren Abstand der Pole von der Berandung des $\Gamma$-Stabilitätsgebiets.

Der Entwurf erfolgt in den folgenden Schritten:

1. Wähle ein Stabilitätsgebiet $\tilde{\Gamma}$, welches alle Eigenwerte aller gewählten Repräsentanten $\boldsymbol{q} \in Q$ möglichst eng umschließt. 
2. Maximiere das Minimum von $a_{0}(\boldsymbol{q}, \boldsymbol{k}), a_{n}(\boldsymbol{q}, \boldsymbol{k})$ und $\Delta_{n-1}(\boldsymbol{q}, \boldsymbol{k})$ bezüglich $\boldsymbol{k}$. Dabei nimmt $\boldsymbol{q}$ die Werte aller gewählten Repräsentanten an.

Ist für dieses neue $\boldsymbol{k}$ die Eigenwertlage aller betrachteten Repräsentanten noch nicht befriedigend, so wird iterativ gemäß Schritt 1 und 2 das $\Gamma$-Stabilitätsgebiet kontrahiert und ein neuer Wert für $\boldsymbol{k}$ gefunden, bis das Ergebnis zufriedenstellend ist. Schließlich ist noch die Robustheit des erhaltenen Reglers für den gesamten Betriebsbereich $Q$ zu überprüfen [12].

\subsection{Stabilitätsradien}

Der Stabilitätsradius ist definiert als die größte reelle Zahl $\rho$, so daß das Polynom $p(s, \boldsymbol{q})$ $\Gamma$-stabil ist für alle $\boldsymbol{q}$ mit $\left\|\boldsymbol{q}^{*}\right\|<\rho$. Dabei handelt es sich bei $\boldsymbol{q}^{*}$ um einen normierten Parametervektor, dessen Elemente

$$
q_{i}^{*}=\frac{q_{i}-q_{i}^{0}}{\alpha_{i}}
$$

auf die entsprechenden Elemente des nominalen Betriebspunktes $\boldsymbol{q}^{0}$ bezogen sind und mit geeigneten Größen $\alpha_{i}$ skaliert und dimensionslos gemacht werden. Der Stabilitätsradius kann für verschiedene Normen und Klassen von Polynomfamilien berechnet werden. Die Ermittlung des Stabilitätsradius ist lediglich ein Analyse-, aber kein Entwurfsverfahren. Der Vorteil ist jedoch, daß er eine differenziertere Aussage liefert als die reine Beantwortung der Frage, ob ein System robust $\Gamma$-stabil ist oder nicht.

\section{Architektur und Bedienung der Toolbox PARADISE}

Die Methoden, wie sie im letzten Abschnitt vorgestellt wurden, erfordern sowohl numerische als auch symbolische Berechnungen: Beispielsweise können Baumstrukturen ausschließlich durch Computer-Algebraprogramme ausgewertet werden, wohingegen die schnelle Konstruktion von Wertemengen nur mit numerischen Programmen machbar ist. Der Benutzer sollte nicht mit der Aufgabe beschäftigt werden, welche Teile der Methoden von numerischen oder symbolischen Programmen ausgeführt werden. Des weiteren erwartet der Benutzer einen einfachen Umgang mit der Toolbox, d.h. die Ein-/Ausgabe sollte über moderne graphische Benutzerschnittstellen (GUI, graphical user interface) und nicht über umständliche Tastatureingabe in Kommandozeilen erfolgen. Eine Programmarchitektur, die große Freiheiten für die Integration verschiedener Berechnungsverfahren läßt, ist in Bild 2 dargestellt. Der Benutzer kommuniziert mit der Toolbox PARADISE über ein GUI, welches in Matlab implementiert ist. Von hier aus können die erforderlichen symbolischen und numerischen Algorithmen ausgeführt werden. Diese Konfiguration bietet einige Vorteile:

- Bei Matlab handelt es sich um ein weitverbreitetes und vielen Regelungstechnikern vertrautes Werkzeug, dessen Syntax leicht verständlich ist. 
- Seit Version 4.0 ist die Möglichkeit GUIs zu programmieren, in Matlab aufgenommen worden.

- Symbolische Berechnungen können entweder über die auf Maple aufbauende Extended Symbolic Toolbox [13] oder durch die Mathematica Symbolic Toolbox für MATLAB [14] ausgeführt werden.

- Teile der numerischen Berechnungen werden durch Matlab selbst ausgeführt, insbesondere, wenn entsprechende Funktionen für bestimmte Aufgaben, z.B. die Berechnung von Wurzelortskurven bereits vorhanden sind. Die Berechnung von $\Gamma$ Stabilitätsgrenzen in einer Parameterebene oder die Algorithmen zur Konstruktion von Wertemengen sind jedoch wesentlich effektiver, wenn sie als kompilierte Programme ausgeführt werden. Übersetzter Fortran- oder $\mathrm{C} / \mathrm{C}++-$ Code kann in Form von MEX-Dateien (Matlab Executables) dazugebunden werden [15].

\subsection{Eingabe}

Die erste Aufgabe des Benutzers bei der Bearbeitung einer Problemstellung der robusten Regelungstechnik mit der Toolbox PARAdise ist die Spezifikation des Problems. Dazu gehören die Eingabe der Strecke, die Annahme einer Reglerstruktur, die Festlegung eines Betriebsbereichs und des $\Gamma$-Gebiets, sowie die Auswahl geeigneter Methoden zum Entwurf und zur Robustheitsanalyse.

\subsubsection{Modellierung einer parametrischen Regelstrecke}

Simulink ist als Aufsatz auf Matlab besonders für Modellierung und Simulation geeignet. Die Toolbox PARADISE verwendet Simulink als eine von mehreren Möglichkeiten zur einfachen Eingabe eines parametrischen Modells der betrachteten Strecke. Simulink bietet eine graphische Benutzeroberfläche, um Systeme in Form von Blockschaltbildern einzugeben. Die Funktion slinmod (Bestandteil des symbolischen Teils von PARADISE) ist in der Lage, eine symbolische Zustandsdarstellung des Regelkreises aus dem Simulink-Modell zu erzeugen $^{3}$. Dieses parametrische Zustandsmodell ist Ausgangspunkt für die in Abschnitt 2 beschriebenen Methoden.

\subsubsection{Eingabe des $\Gamma$-Stabilitätsgebietes}

PARADISE verfügt über einen grafischen Editor („Г-Editor“) zur Konstruktion von $\Gamma$ Berandungen in der Eigenwertebene. Der Benutzer kann verschiedene Basiselemente aus

\footnotetext{
${ }^{3}$ Es gibt eine Simulink-Funktion linmod, die ein linearisiertes Zustandsmodell eines gegebenen SimulinkModells berechnet. Diese Funktion ist für parametrische Modelle jedoch nicht brauchbar, da nur ein numerisches lineares Modell für einen nominalen Betriebspunkt erzeugt werden kann und somit alle Parameter festgelegt werden müssen, bevor linmod ausgeführt werden kann.
} 
einer Bibliothek miteinander kombinieren. Diese Bibliothek besteht gegenwärtig aus folgenden Elementen: Eine Gerade mit konstantem Realteil (Bild 1 a)), ein zur reellen Achse paralleles Geradenpaar mit konjugiert komplexem Imaginärteil, ein Geradenpaar konstanter Dämpfung (Bild 1 b)), eine Hyperbel, Kreise (Bild 1 c)) und Ellipsen. Die geometrischen Parameter aller Basiselemente können angegeben und später jederzeit modifiziert werden. Dies kann entweder über ein entsprechendes Eingabefenster geschehen oder durch Anklicken und Verschieben des Basiselements mit der Maus im $\Gamma$-Editor.

Die Basiselemente können auch invertiert werden bzgl. des $\Gamma$-stabilen Gebiets, welches sie beranden. Beispielsweise ist als Voreinstellung das Innere des Basiselements „Kreis“ als $\Gamma$-stabil definiert und das Äußere $\Gamma$-instabil, bei Inversion umgekehrt. Die Basiselemente können beliebig miteinander logisch verknüpft werden, so daß jede Schnitt- und Vereinigungsmengenkombination der Eigenwertmengen möglich wird, die durch die einzelnen Basiselemente als $\Gamma$-stabil definiert sind. Derzeit können bis zu 10 Basiselemente miteinander kombiniert werden, wodurch sich sämtliche $\Gamma$-Berandungen konstruieren lassen, die in der Praxis der robusten parametrischen Regelungstechnik sinnvoll sind. Bild 3 zeigt das Beispiel eines $\Gamma$-Gebietes, das aus einem Geradenpaar konstanter Dämpfung, zwei konzentrischen Kreisen mit Mittelpunkt im Ursprung und einem Paar konjugiert komplexer Ellipsen aufgebaut wurde. Der Kreis mit dem kleineren Radius wurde invertiert und mit dem größeren Kreis sowie dem Geradenpaar geschnitten. Das so erhaltene Kreisringsegment wurde mit dem Inneren der Ellipsen zum als $\Gamma$-stabil definierten $\Gamma$-Gebiet vereinigt. Dies könnte z.B. in einem praktischen Anwendungsfall sinnvoll sein, bei dem 2 konjugiert komplexe Eigenwerte innerhalb der Ellipsen, die restlichen Eigenwerte innerhalb des Kreisringsegments verbleiben sollen. Dieses wird durch den großen Kreis in seiner Bandbreite und durch das Geradenpaar bzgl. einer Minimaldämpfung beschränkt. Der kleine Kreis soll verhindern, daß das Systemverhalten zu langsam wird. Die Anteile der Basiselemente, die schließlich zur $\Gamma$-Berandung beitragen, werden durch den $\Gamma$-Editor berechnet, als durchgezogene Linien gezeichnet und von Schraffur umgeben, die den $\Gamma$-instabilen Bereich markiert. Die Bestimmung der relevanten Basiselement-Anteile ist auch für die spätere Anwendung der Methoden aus Abschnitt 2 erforderlich. Mit dem $\Gamma$-Editor kann nicht nur eines, sondern eine ganze Menge aus mehreren $\Gamma$-Gebieten erstellt und bearbeitet werden. Einzelne $\Gamma$-Gebiete aus dieser Menge können später unterschiedlichen Betriebspunkten zugeordnet werden, siehe folgender Abschnitt.

\subsubsection{Eingabe des Betriebsbereichs}

Wie in Abschnitt 3.1.1 bereits beschrieben, werden nach der Eingabe des Modells die darin enthaltenen Parameter durch PARADISE automatisch erkannt. Nun kann der Benutzer klassifizieren, welche dieser Größen Reglerparameter, veränderliche ${ }^{4}$ Parameter oder konstante Parameter sind. Als Voreinstellung werden alle Größen, deren Name mit dem Buchstaben $\mathrm{k}$ beginnt als Reglerparameter, die mit $\mathrm{q}$ beginnen als veränderliche Parameter und die

\footnotetext{
${ }^{4}$ Unter veränderlichen Parametern werden hier sowohl unsichere als auch variierende, aber meßbare Parameter zusammengefaßt.
} 
übrigen als konstante Parameter klassifiziert. Schließlich müssen die numerischen Werte der konstanten Parameter und Reglerparameter bzw. die Grenzen des Betriebsbereichs der veränderlichen Parameter angegeben werden. Dies geschieht im linken bzw. mittleren Teil des Eingabefensters, das in Bild 4 beispielhaft für das in Abschnitt 4 folgende Anwendungsbeispiel gezeigt ist. Im mittleren Teil wird auch die Anzahl der Rasterpunkte für Verfahren, bei denen Rasterung der veränderlichen Parameter erforderlich ist, festgelegt. Der rechte Teil des Eingabefensters erlaubt die Festlegung einer beliebigen Anzahl von Betriebspunkten z.B. als Repräsentanten für den Reglerentwurf oder für ein Multi-ModellProblem. Jedem dieser Betriebspunkte kann bei Bedarf ein eigenes $\Gamma$-Gebiet zugeordnet werden, so daß für unterschiedliche Betriebspunkte unterschiedlichen Anforderungen an die Systemdynamik Rechnung getragen werden kann. Im gezeigten Beispiel hat der Benutzer zwei Repräsentanten definiert, von denen der erste dargestellt ist. Diesem ist das $\Gamma$-Gebiet mit der Nummer 2 zugeordnet.

\subsection{Ausgabe}

Die Ergebnisdarstellung ist von den gewählten Entwurfs- und Analyseverfahren abhängig. Das Hauptziel ist die verständliche Präsentation dieser Ergebnisse, d.h. wenn immer möglich, wird eine grafische Ausgabe bevorzugt.

Im folgenden Abschnitt wird anhand eines Beispiels der Umgang mit PARADISE erläutert.

\section{Anwendung: Entwurf eines Spurführungsreglers in einer Invarianzebene}

Die Automatisierung des Straßenverkehrs, wie sie beispielsweise im kalifornischen PATHProgramm [16] untersucht wird, erfordert im hohen Maße den Einsatz von Regelung. Eine wesentliche Teilkomponente hierzu ist die seitliche Spurführung von Straßenfahrzeugen. Die Aufgabe besteht darin, die Abweichung von der Sollspur möglichst gering zu halten und gleichzeitig entsprechenden Komfort für die Passagiere zu gewährleisten. Die Regelung muß bei allen zulässigen Fahrgeschwindigkeiten $v$ robust sein gegenüber unterschiedlichem

Kraftschlußkoeffizienten $\mu$ zwischen Reifen und Straße und Änderungen in der Masse des Fahrzeugs z.B. durch Passagiere und Zuladung.

Eine Skizze eines spurgeführten Fahrzeugs ist in Bild 5 dargestellt. Hierbei wurde das Einspurmodell verwendet, welches man erhält, wenn die Räder jeweils einer Achse vereinfachend zu einem Rad in der Mitte der Achse zusammengefaßt werden werden [17]. Eine linearisierte Zustandsdarstellung des spurgeführten Fahrzeugs lautet 


$$
\left[\begin{array}{l}
\dot{y}_{v} \\
\ddot{y}_{v} \\
\dot{y}_{h} \\
\ddot{y}_{h}
\end{array}\right]=\left[\begin{array}{cccc}
0 & 1 & 0 & 0 \\
d_{21} & d_{22} & -d_{21} & d_{24} \\
0 & 0 & 0 & 1 \\
d_{41} & d_{42} & -d_{41} & d_{44}
\end{array}\right]\left[\begin{array}{l}
y_{v} \\
\dot{y}_{v} \\
y_{h} \\
\dot{y}_{h}
\end{array}\right]+\left[\begin{array}{cc}
0 & 0 \\
b_{2} & -v^{2} \\
0 & h_{4} v \\
b_{4} & -v^{2}
\end{array}\right]\left[\begin{array}{c}
\delta_{v} \\
\rho_{r e f}
\end{array}\right]
$$

mit

$$
\begin{aligned}
d_{21} & =\frac{-\ell_{s v} h_{1}}{\tilde{J} h_{4}}+\frac{h_{2}}{\tilde{m} h_{4}} & d_{41} & =\frac{\ell_{s h} h_{1}}{\tilde{J} h_{4}}+\frac{h_{2}}{\tilde{m} h_{4}} \\
d_{22} & =\frac{\ell_{s v}\left(\ell_{s h} h_{1}-h_{3}\right)}{\tilde{J} h_{4} v}-\frac{\ell_{s h} h_{2}-h_{1}}{\tilde{m} h_{4} v} & d_{42} & =\frac{\ell_{s h}\left(h_{3}-\ell_{s h} h_{1}\right)}{\tilde{J} h_{4} v}-\frac{\ell_{s h} h_{2}-h_{1}}{\tilde{m} h_{4} v} \\
d_{24} & =\frac{\ell_{s v}\left(h_{3}+\ell_{s v} h_{1}\right)}{\tilde{J} h_{4} v}-\frac{\ell_{s v} h_{2}+h_{1}}{\tilde{m} h_{4} v} & d_{44} & =\frac{-\ell_{s h}\left(h_{3}+\ell_{s v} h_{1}\right)}{\tilde{J} h_{4} v}-\frac{\ell_{s v} h_{2}+h_{1}}{\tilde{m} h_{4} v} \\
b_{2} & =h_{6}+\ell_{s v} h_{5} & b_{4} & =h_{6}-\ell_{s h} h_{5}
\end{aligned}
$$

unter Verwendung der Hilfsgrößen

$$
\begin{array}{ll}
h_{1}=c_{h} \ell_{h}-c_{v} \ell_{v} & h_{2}=c_{v}+c_{h} \\
h_{3}=c_{h} \ell_{h}^{2}+c_{v} \ell_{v}^{2} & h_{4}=\ell_{s v}+\ell_{s h} \\
h_{5}=c_{v} \ell_{v} / \tilde{J} & h_{6}=c_{v} / \tilde{m}
\end{array}
$$

Hierbei ist $\tilde{m}=m / \mu$ die (unsichere) virtuelle Masse, $\tilde{J}=J / \mu$ das (unsichere) virtuelle Gierträgheitsmoment, $c_{v}$ und $c_{h}$ sind die Schräglaufsteifigkeiten der Reifen. Die Abstände der Achsen in Längsrichtung $\ell_{v}$ und $\ell_{h}$ beziehen sich auf den Schwerpunkt, $\ell_{s v}$ und $\ell_{s h}$ sind die entsprechenden Abstände der Sensoren. Diese messen die seitlichen Abweichungen $y_{v}$ und $y_{h}$ von der Sollspur, welche z.B. durch ein elektrisches Leitkabel oder magnetische Markierungen in der Fahrbahn vorgegeben wird. Der Betrag des Geschwindigkeitsvektors im Fahrzeugschwerpunkt $\vec{v}$ ist $v$. Eingangsgrößen sind der Vorderradlenkwinkel $\delta_{v}$ und die Sollspurkrümmung $\rho_{\text {ref }}$.

Ein mögliches Spurführungskonzept wurde in [1] vorgestellt. Es basiert auf einer vollständigen Zustandsvektorrückführung

$$
\delta_{v}=-\boldsymbol{k}\left[\begin{array}{llll}
y_{v} & \dot{y}_{v} & y_{h} & \dot{y}_{h}
\end{array}\right]^{T}
$$

auf den Vorderradlenkwinkel. Es muß nun ein Rückführvektor $\boldsymbol{k}$ ermittelt werden, der das Fahrzeug für den gesamten Betriebsbereich robust $\Gamma$-stabilisiert.

Das in Abschnitt 2.1 vorgestellte Parameterraumverfahren ist hierfür nicht unmittelbar geeignet, da die Dimension von $\boldsymbol{k}$ größer als zwei ist. Es bietet sich jedoch ein Entwurf in einer Invarianzebene an, wie in Abschnitt 2.2 beschrieben. Das System (8) ist vierter Ordnung und hat neben zwei Eigenwerten im Ursprung (Integratoren für die Abstände $y_{v}$ und $y_{h}$ ) noch die beiden Eigenwerte aus der Fahrdynamik des Einspurmodells. Diese sind für den gesamten Betriebsbereich $\Gamma$-stabil und genügend gedämpft. Es liegt also nahe, die bereits $\Gamma$-stabilen Eigenwerte in ihrer ursprünglichen Position zu belassen und lediglich die beiden Eigenwerte im Ursprung in die linke Halbebene zu verschieben. Für die Berechnungen 
werden die Daten bzw. der Betriebsbereich für das Einspurmodell eines BMW 735i verwendet. Die Dämpfung des geregelten Systems soll mindestens 0.4 betragen. Um zu langsame Zeitantworten zu verhindern, soll der maximale Realteil auf -0.5 beschränkt werden. Beide Forderungen werden durch die Hyperbel

$$
\frac{\sigma^{2}}{0.25}-\frac{\omega^{2}}{1.3125}=1
$$

erfüllt, die die Berandung des $\Gamma$-stabilen Gebiets in der Eigenwertebene $s=\sigma+j \omega$ beschreibt (siehe Bild 8, rechts oben).

Da es nicht ohne weiteres möglich ist, in einem Entwurfsschritt einen Regler zu ermitteln, der den gesamten, in Bild 6 dargestellten Betriebsbereich $\Gamma$-stabilisiert, soll zunächst versucht werden, eine endliche Anzahl von Repräsentanten simultan zu $\Gamma$-stabilisieren. In diesem Beispiel werden hierzu die vier Eckpunkte $\boldsymbol{q}_{1}$ bis $\boldsymbol{q}_{4}$ gewählt.

Würde man nun versuchen, das Entwurfsverfahren in der Invarianzebene auf das Modell mit den in (8) gegebenen Systemgleichungen anzuwenden, so würde dies bei der Implementierung des Reglers im realen Fahrrzeug sicherlich nicht zum gewünschten Erfolg führen. Der Grund ist, daß der Aktuator zum Aufbringen des erforderlichen Lenkwinkels $\delta_{v}$ vernachlässigt wurde. Die Dynamik dieses Aktuators schränkt die Reglerauswahl jedoch erheblich ein.

Aus diesem Grund wird ein zweites Modell hinzugezogen, welches das bisherige Modell um einen Aktuator erweitert, wobei für diesen das Übertragungsverhalten ähnlich einer Butterworth-Pol-Konfiguration dritter Ordnung (keine Nullstellen) mit einer Bandbreite von $10 \mathrm{~Hz}$ angenommen wird. Das dann resultierende Simulink-Modell ist in Bild 7 rechts oben dargestellt. Das Fenster links oben ist das Hauptkontrollfenster von PARADISE (das Logo stellt übrigens die farbkodierte Wertemenge der Gierdynamik eines Fahrzeugs dar).

Aus Bild 7 ist eine weitere Eigenschaft der Toolbox PARADISE ersichtlich: Um nicht alle Koeffizientenfunktionen in Simulink angeben zu müssen, besteht die Möglichkeit, die Definition des Modells in Simulink sehr allgemein zu halten. So wurden beispielsweise im Block Vehicle die Koeffizienten $d_{i j}$ und $b_{i}$ lediglich in dieser Abkürzung angegeben. Das Simulink-Modell wird beim Einlesen in PARADISE analysiert und sämtliche auftauchenden symbolischen Parameter werden als solche erkannt. Für diese Parameter kann der Benutzer anschließend einen beliebigen Ausdruck angeben, der dann substituiert wird. In Bild 7 ist links unten das hierfür erforderliche Fenster mit den tatsächlichen Abhängigkeiten der Koeffizienten $d_{i j}$ und $b_{i}$ von den Fahrzeugparametern dargestellt. Wird nun der Aktuator mitberücksichtigt, kann das Verfahren des Entwurfs in der Invarianzebene nicht mehr unmittelbar angewandt werden, da die Aktuatorzustände nicht gemessen und zurückgeführt werden können. Geht man jedoch näherungsweise davon aus, daß die Aktuatordynamik die Dynamik des Gesamtsystems nicht allzu gravierend verändert, so ist es ein gangbarer und sinnvoller Weg, zunächst die Invarianzebene für das System ohne Aktuator zu ermitteln und schließlich die $\Gamma$-Stabilitätsgrenzen des Systems mit Aktuator in dieser Invarianzebene zu bestimmen. 
Diese Vorgehensweise kann mit der Toolbox PARADISE angewandt werden. In Bild 8 ist links oben das Kontrollfenster für den Entwurf in der Invarianzebene zu sehen. Es soll die Invarianzebene für den Mittelpunkt des gegebenen Betriebsbereichs für das System bmwinv1 (Simulink-Modell ohne Aktuator) berechnet werden. Rechts im Bild ist das gewünschte $\Gamma$-Gebiet mit den Eigenwerten des ungeregelten Systems ohne Aktuator dargestellt. Durch Anklicken der Eigenwerte teilt der Anwender der Toolbox PARAdISE mit, welche Eigenwerte verschoben werden sollen, in diesem Fall die beiden Eigenwerte im Ursprung. Anschließend kann die Berechnung der $\Gamma$-Stabilitätsgrenzen in der dadurch festgelegten Invarianzebene für das System mit Aktuator (bmwinv2) erfolgen. Das Ergebnis ist in Bild 8 im Fenster links unten zu sehen, in dem in einem Ausschnitt der $\left(\kappa_{a}, \kappa_{b}\right)$ Invarianzebene die $\Gamma$-Stabilitätsgrenzen aller vier Repräsentanten $\boldsymbol{q}_{1}$ bis $\boldsymbol{q}_{4}$ dargestellt sind. Diese Grenzen unterteilen die $\left(\kappa_{a}, \kappa_{b}\right)$-Ebene in eine endliche Anzahl von Gebieten. Durch Auswahl beliebiger Punkte in der $\left(\kappa_{a}, \kappa_{b}\right)$-Ebene mit dem Maus-Cursor kann der Anwender prüfen, ob diese Punkte simultan $\Gamma$-stabilisierend sind oder nicht. So kann er herausfinden, ob es ein Gebiet simultan $\Gamma$-stabilisierender Reglerparameter gibt und falls ja, wo dieses liegt. Aus diesem Gebiet muß der Benutzer einen Regler auswählen, der dann markiert wird.

Für das Anwendungsbeispiel existiert eine Menge von Reglern, die alle Repräsentanten gleichzeitig $\Gamma$-stabilisiert. Der daraus ausgewählte Regler ist in der $\left(\kappa_{a}, \kappa_{b}\right)$-Ebene von Bild 8 mit einem „,+ "markiert. Durch die simultane $\Gamma$-Stabilisierbarkeit ist keine Betriebsanpassung der Reglerparameter durch die Geschwindigkeit notwendig. Um eine bessere Regelgüte für unterschiedliche Geschwindigkeiten erzielen zu können, wird allerdings eine Betriebsanpassung sinnvoll sein, dies wird hier aus Gründen der Kürze jedoch nicht illustriert. Eine Möglichkeit wäre hier, für jeweils eine feste Geschwindigkeit $v_{0}$ einen Regler in einer entsprechend gewählten Invarianzebene für die beiden Repräsentanten $\left[\begin{array}{ll}v_{0} & \tilde{m}^{-}\end{array}\right]^{T}$ und $\left[\begin{array}{ll}v_{0} & \tilde{m}^{+}\end{array}\right]^{T}$ zu bestimmen und die daraus für mehrere Geschwindigkeiten ermittelten Reglerkoeffizienten über den gesamten Geschwindigkeitsbereich zu interpolieren.

In einer nachfolgenden Stabilitätsanalyse muß auf jeden Fall sichergestellt werden, daß der gewählte Regler das gesamte Kontinuum des Betriebsbereichs $\Gamma$-stabilisiert. Diese Analyse kann beispielsweise mit dem Parameterraumverfahren durchgeführt werden, wobei sinnvollerweise die $\Gamma$-Stabilitätsgrenzen des geregelten Systems in der $(v, \tilde{m})$-Ebene berechnet werden.

\section{$5 \quad$ Zusammenfassung}

Ein Nachteil der Methoden der robusten Regelung, die auf einem physikalisch motivierten Modell basieren, waren bislang die aufwendigen symbolischen und numerischen Berechnungen, wie sie z.B. für das Parameterraumverfahren notwendig sind. Die hier vorgestellte Toolbox PARADISE überwindet diese Schwierigkeiten, indem sie eine computergestützte Implementierung der Methoden auf eine einfach zu bedienende und flexible Weise anbietet. Gegenwärtig in PARADISE verfügbare Verfahren sind das Parameterraumverfahren, 
der Entwurf in einer Invarianzebene, Wertemengenkonstruktion und Baumstrukturierte Zerlegung, der Entwurf durch Kontraktion eines Polgebiets sowie die Berechnung von Stabilitätsradien.

PARADISE benötigt Matlab und Simulink, welches weitverbreitete Werkzeuge zur Bearbeitung regelungstechnischer Aufgabenstellungen sind. Die symbolischen Berechnungen werden vor dem Anwender verborgen im Hintergrund mit Mathematica oder der Symbolic Toolbox für Matlab ausgeführt.

In einem Anwendungsbeispiel wurde der Entwurf eines Zustandsreglers in einer Invarianzebene für das Spurführungsproblem eines PKW durchgeführt. Hierbei konnte in einer Erweiterung dieses Verfahrens die Dynamik des Aktuators (dessen Zustände nicht meßbar sind) mitberücksichtigt werden.

Informationen über den aktuellen Stand der Toolbox PARADISE sind unter http://www.op.dlr.de/FF-DR-RR/paradise

im World Wide Web abrufbar.

\section{Literatur}

[1] J. Ackermann, A. Bartlett, D. Kaesbauer, W. Sienel, and R. Steinhauser, Robuste Regelung. Analyse und Entwurf von linearen Regelungssystemen mit unsicheren physikalischen Parametern. Berlin: Springer, 1993.

[2] G. Balas, J. Doyle, K. Glover, A. Packard, and R. Smith, The $\mu$ analysis and synthesis toolbox. The MathWorks, 1991.

[3] R. Y. Chiang and M. G. Safonov, Robust control toolbox. The MathWorks, 1992.

[4] R. Frazer and W. Duncan, "On the criteria for the stability of small motions," in Proc. Royal Society A, vol. 124, pp. 642-654, 1929.

[5] J. Ackermann, D. Kaesbauer, and R. Münch, "Robust $\Gamma$-stability analysis in a plant parameter space," Automatica, vol. 27, pp. 75-85, 1991.

[6] W. Sienel, "Design of robust gain scheduling controllers," in Proc. Euraco Robust and Adaptive Control Tutorial Workshop, (Dublin), 1994.

[7] J. Ackermann, Sampled-data control systems: analysis and synthesis, robust system design. Berlin: Springer, 1985.

[8] J. Ackermann and S. Türk, "A common controller for a family of plant models," in Proc. 21st IEEE Conf. Decision and Control, (Orlando), pp. 240-244, 1982.

[9] A. Mikhailov, "Method of harmonic analysis in control theory," Avtomatika $i$ Telemekhanika, vol. 3, pp. 27-81, 1938. 
[10] W. Sienel, Analyse und Entwurf von robusten Regelungssystemen durch Konstruktion von komplexen Wertemengen. Dissertation, Technische Universität München, 1994.

[11] W. Sienel, "On stability profiles," in Proc. 13th IFAC World Congress, (San Francisco), 1996.

[12] D. Kaesbauer, Robuster Reglerentwurf durch Kontraktion eines Polgebiets. Dissertation, Institut für Elektrotechnik, Technische Universität Graz, 1986.

[13] D. Chen and C. Moler, Symbolic Math Toolbox. The MathWorks, 1994.

[14] D. Stein, Mathematica Symbolic Toolbox for Matlab. Wolfram Research, Inc., 1994. freely available on mathsource.wri.com, item \#0205-951.

[15] The MathWorks, External Interface Guide, 1993.

[16] S. E. Shladover et al., "Automatic vehicle control developments in the PATH Program," IEEE Trans. on Vehicular Technology, vol. 40, no. 1, pp. 114-130, 1991.

[17] P. Riekert and T. Schunck, "Zur Fahrmechanik des gummibereiften Kraftfahrzeugs," Ingenieur Archiv, vol. 11, pp. 210-224, 1940. 

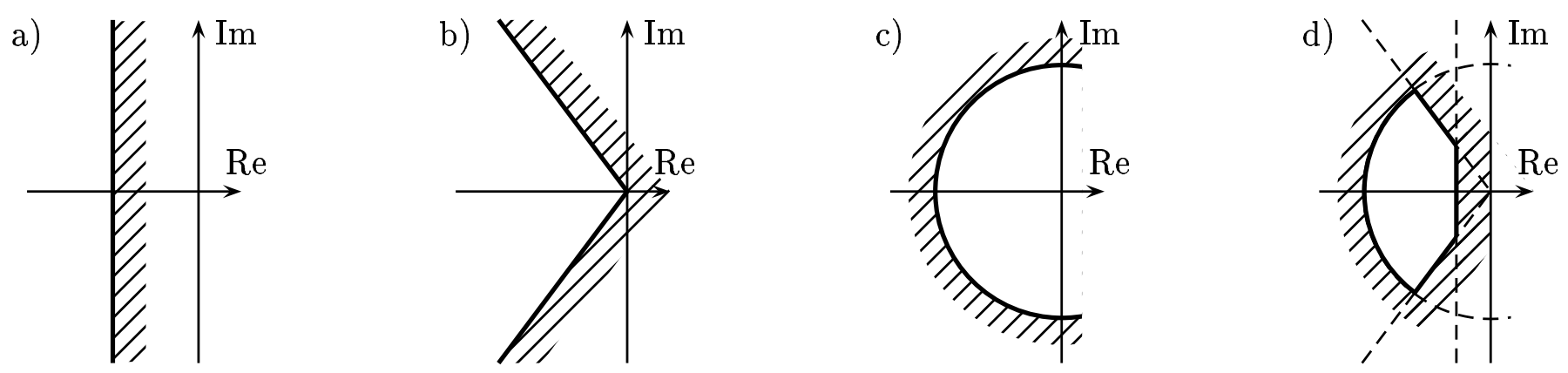

Bild 1: Einige Basiselemente für die Berandung des $\Gamma$-Stabilitätsgebiets (a-c) und deren Schnittmenge (d) 


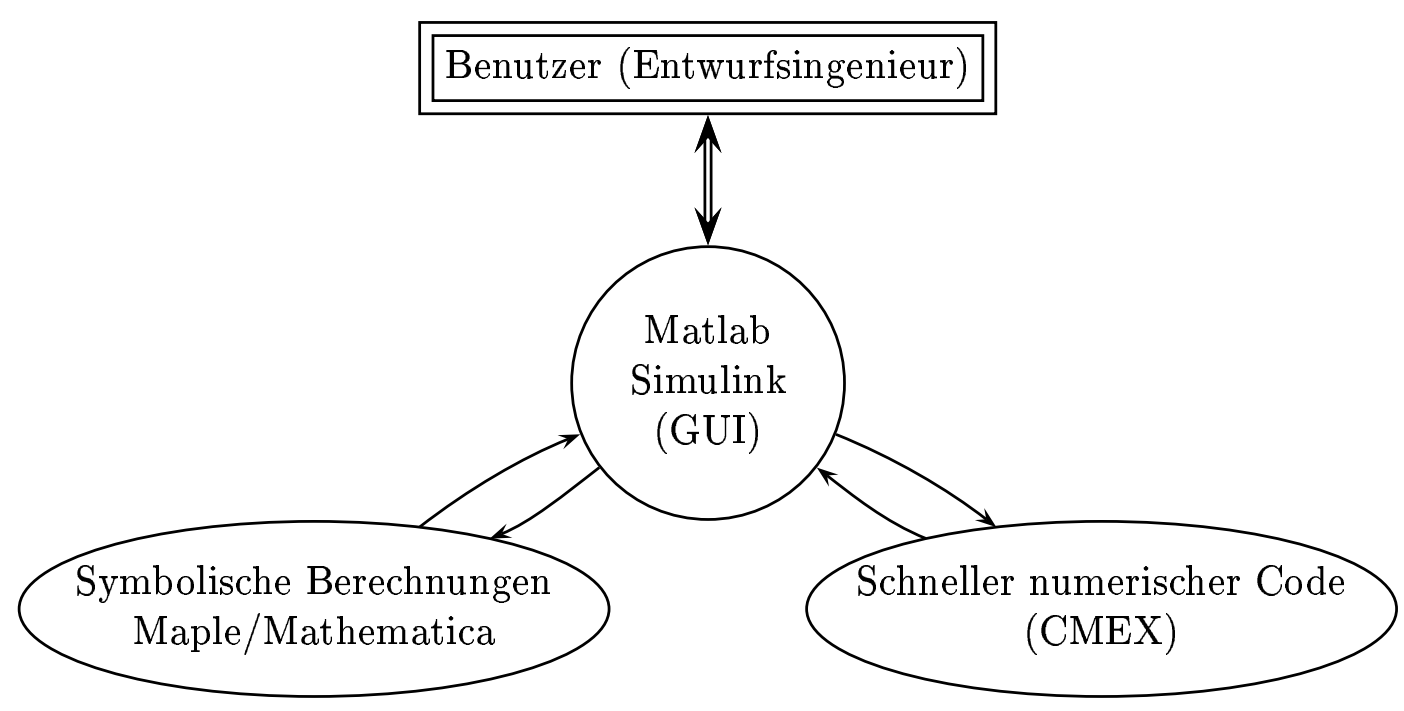

Bild 2: Architektur der Toolbox 


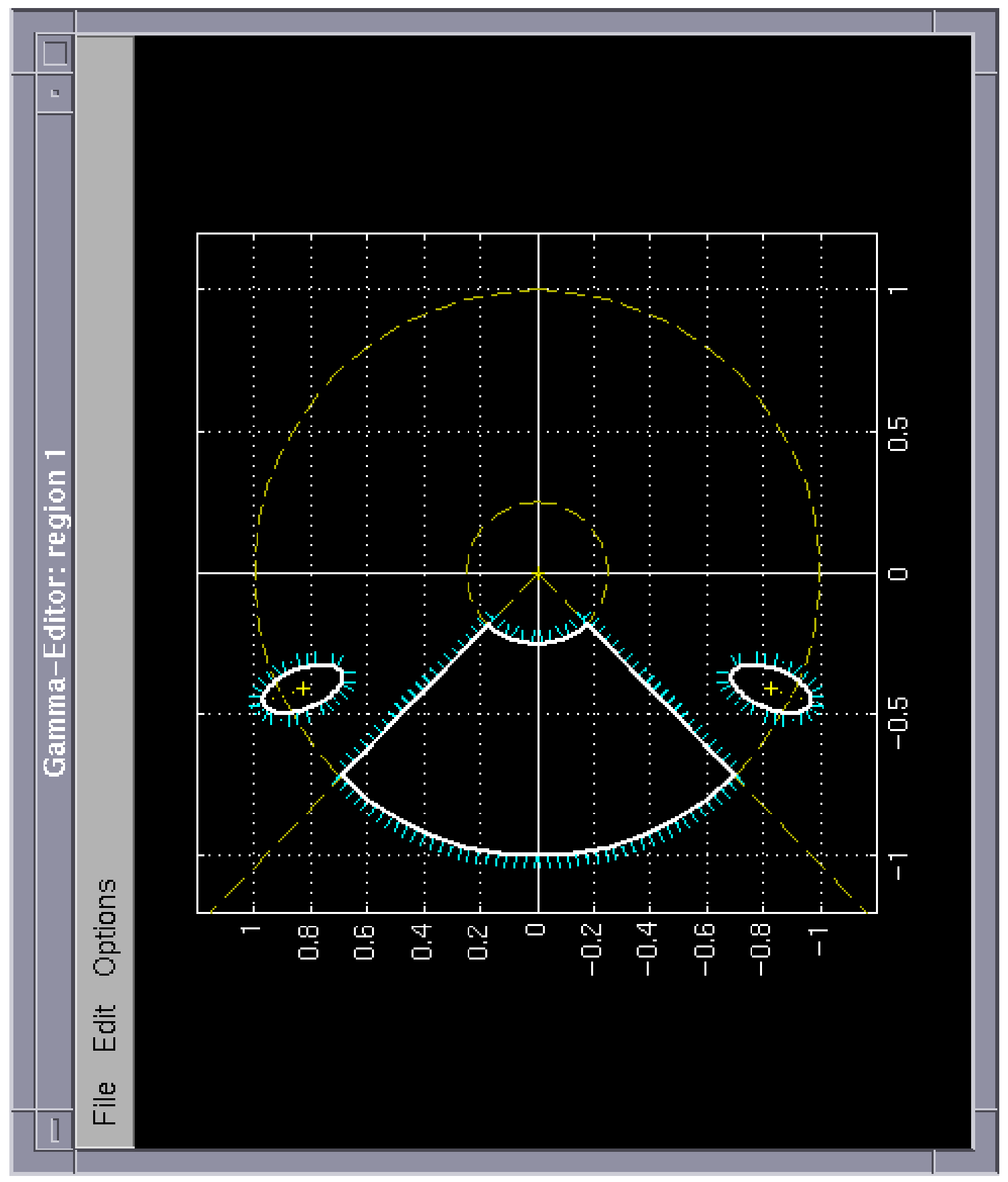

Bild 3: Г-Editor 


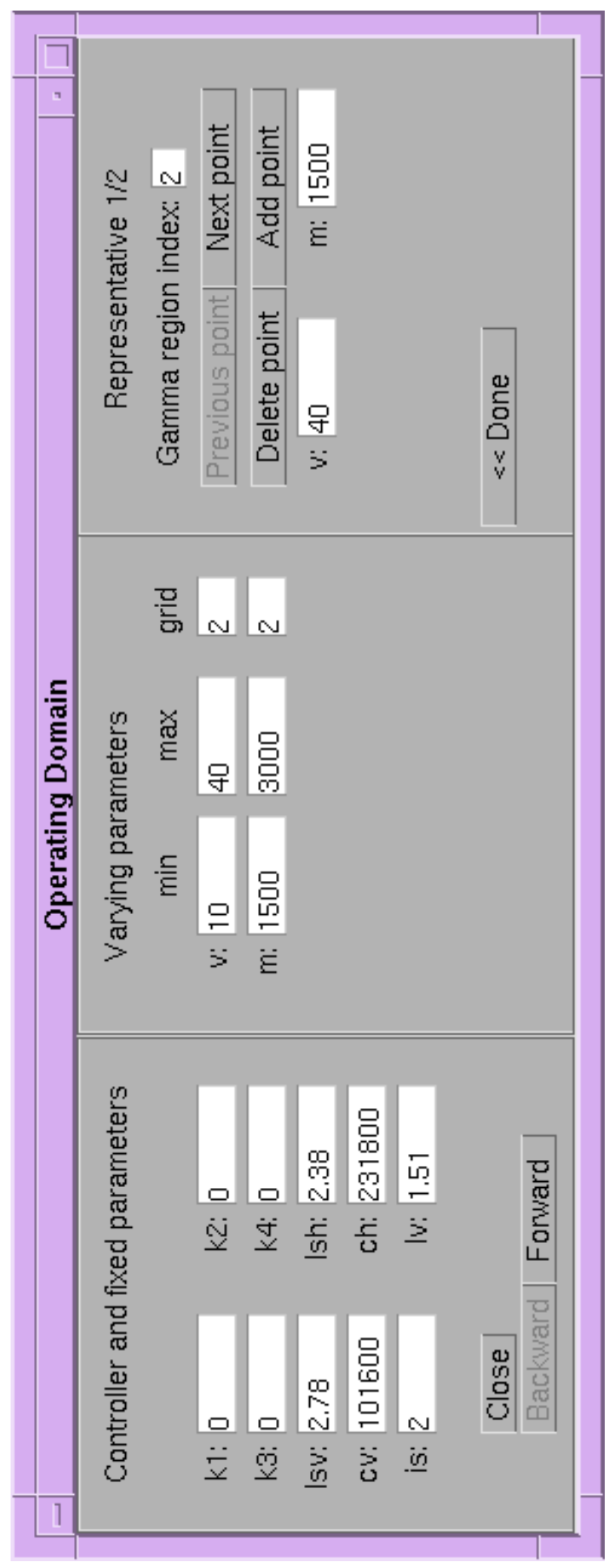

Bild 4: GUI für die Festlegung konstanter Parameter und des Betriebsbereichs 


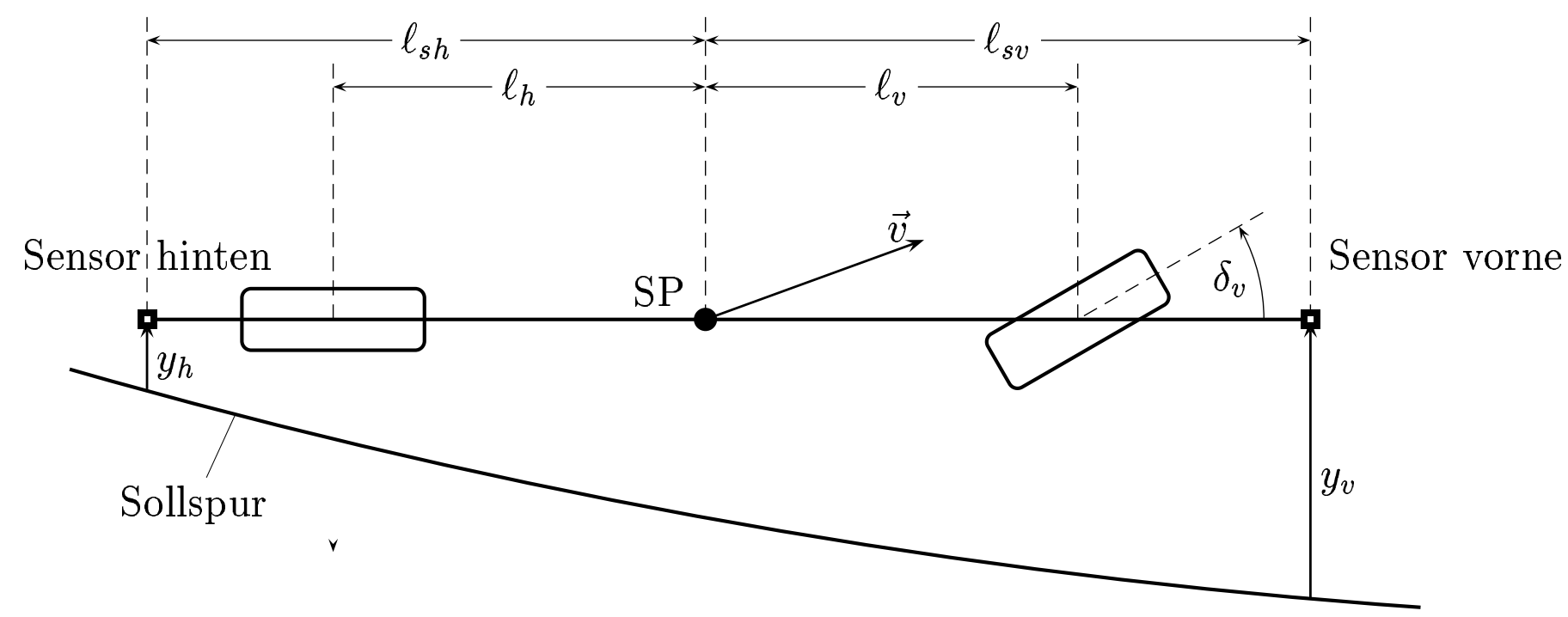

Bild 5: Einspurmodell für die automatische Spurführung

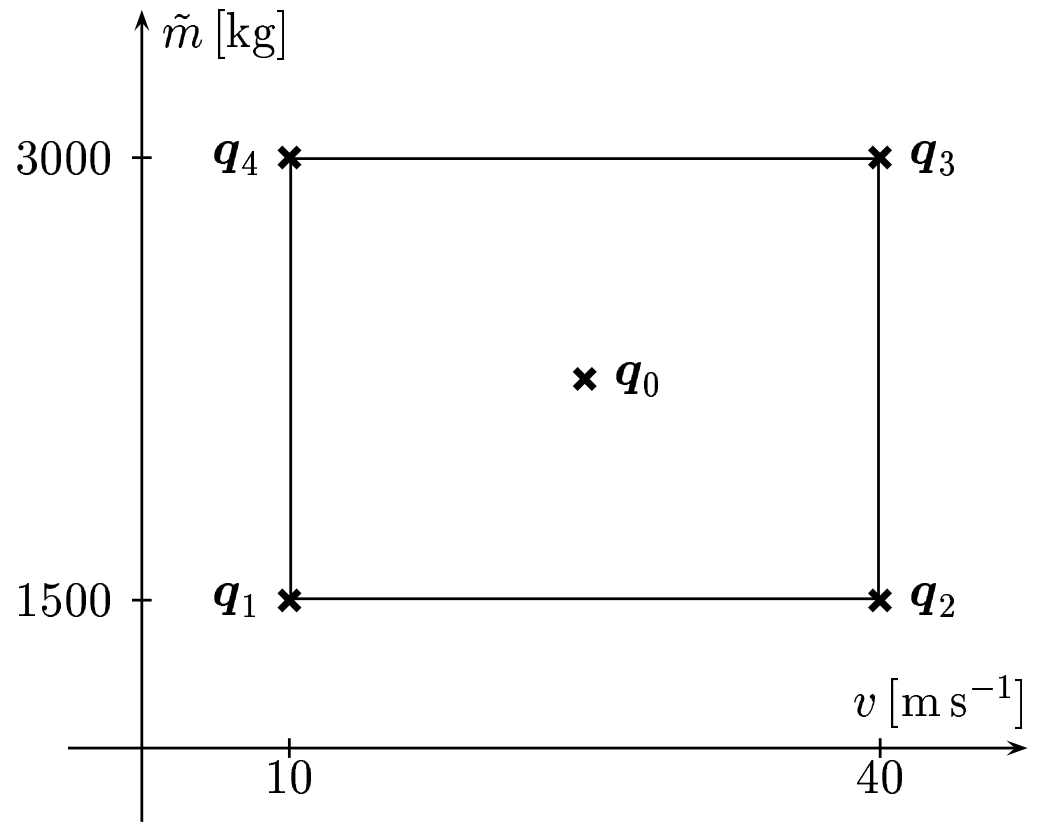

Bild 6: Betriebsbereich des Fahrzeugs mit Repräsentanten 


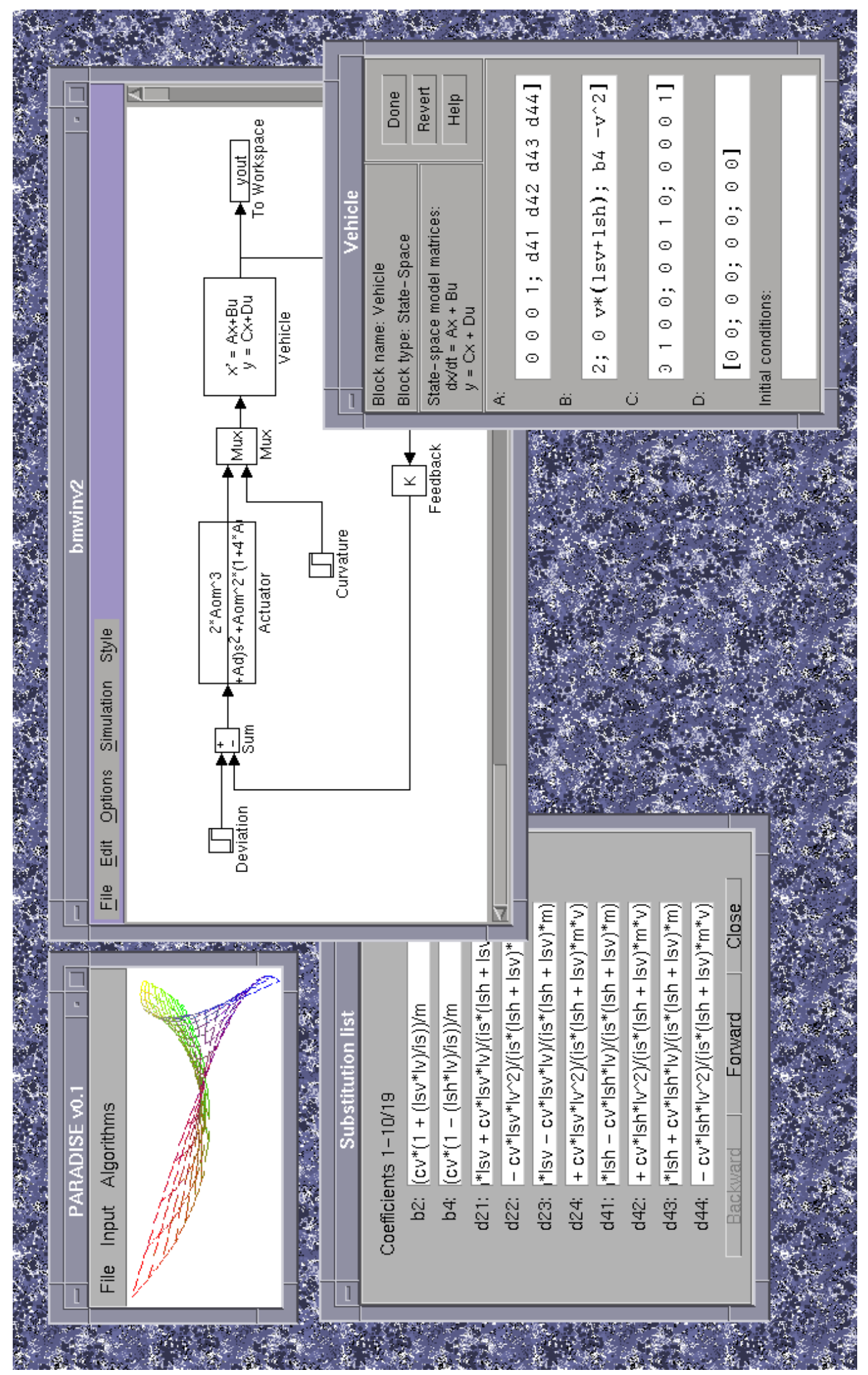

Bild 7: Definition der Strecke 


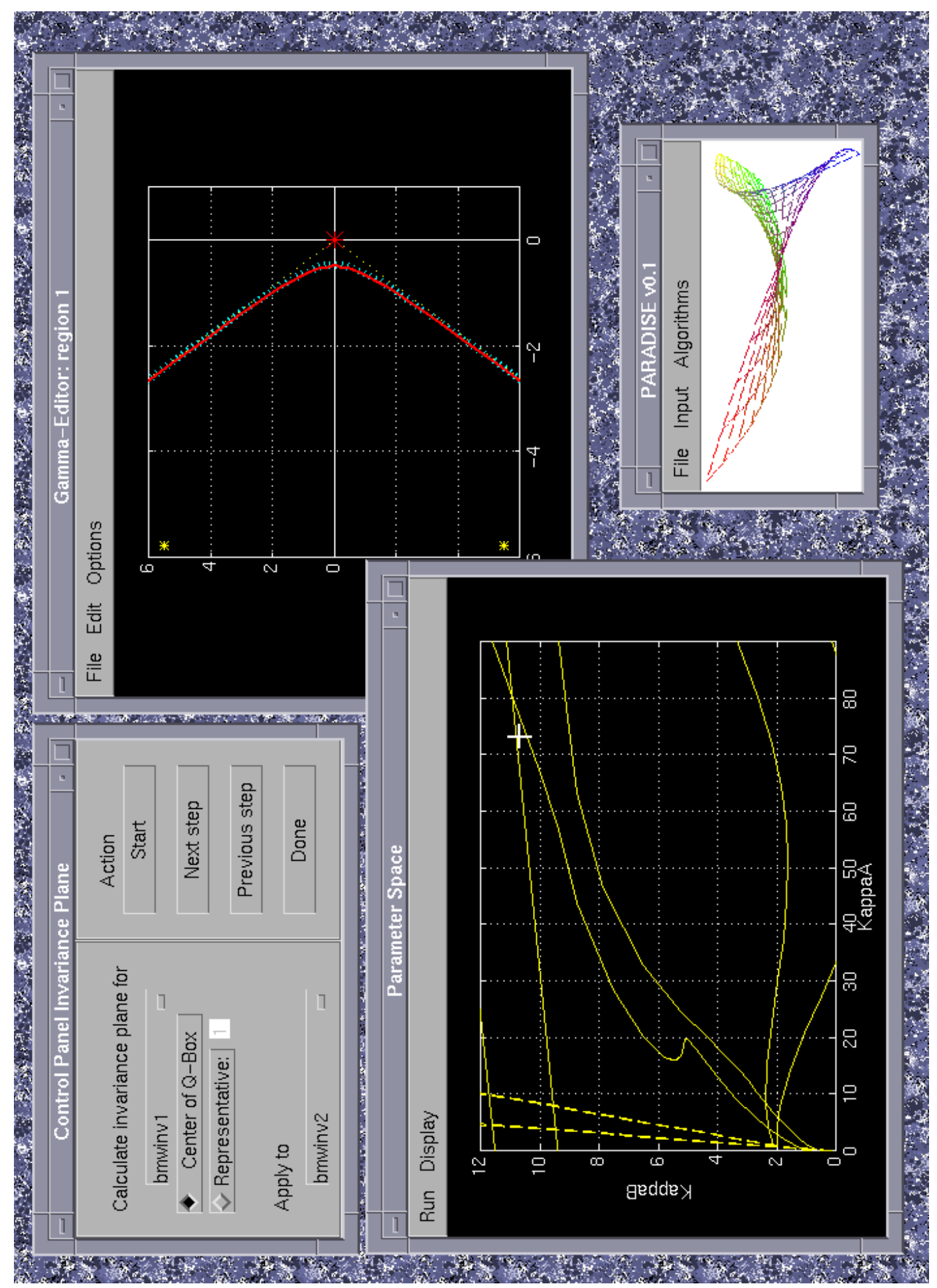

Bild 8: Berechnung der $\Gamma$-Stabilitätsgrenzen in der Invarianzebene 